\title{
Identificação do Perfil Emocional do Aluno Através de Análise de Sentimento: Combatendo a Evasão Escolar
}

\author{
Míria Bobó, Fernanda Campos, Victor Ströele, \\ José Maria N. David, Regina Braga \\ Universidade Federal de Juiz de Fora
}

Programa de Pós-graduação em Ciência da Computação

Rua José Lourenço Kelmer, s/n-Campus Universitário- 36036-900-Juiz de Fora/MG

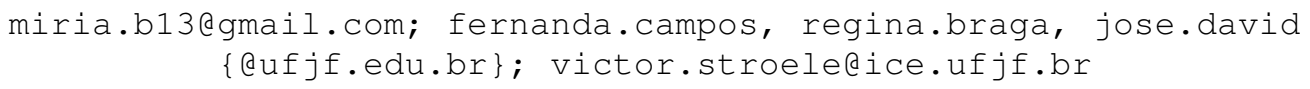

\begin{abstract}
This article presents the SASys architecture, which main goal is to identify the student's emotional profile through the use of FrameNet Brasil to establish the author's feeling in texts. A recommendation system, based on the student's emotional profile and learning style, sends motivational messages to mitigate school dropout. The proposal was evaluated by a case study with students of the Methodology of Scientific and Educational Research class of a learning distance course. The results point to the feasibility of the proposal for the assertiveness of the student's emotional profile during the class and detection of students' risk of dropout.
\end{abstract}

Resumo. Este artigo apresenta a arquitetura SASys, cujo objetivo principal é identificar o estado emocional do aluno, através do uso da FrameNet Brasil para determinar o sentimento do autor em textos. Um sistema de recomendação, baseado no estado emocional do aluno e do seu estilo de aprendizagem, envia mensagens motivacionais para mitigar a evasão. A proposta foi avaliada com a condução de um estudo de caso com alunos da disciplina de Metodologia de Pesquisa Científica e Educacional de um curso à Distância. Os resultados apontam para a viabilidade da proposta para a assertividade do estado emocional do aluno ao longo da disciplina e detecção de alunos com risco de evasão.

\section{Introdução}

Nas redes sociais as pessoas compartilham livremente suas opiniões, experiências e sentimentos sobre produtos, serviços e acontecimentos das mais variadas esferas da vida social [Bamakan et al. 2019]. A Análise de Sentimento (AS) é um conjunto de técnicas que procuram identificar o sentimento do autor em relação ao texto escrito por ele [Altrabsheh et al. 2014]. Na educação a AS visa diminuir os aspectos que influenciam, negativamente, a motivação e o rendimento dos alunos [Lu et al. 2015].

Os Ambientes Virtuais de Aprendizagem (AVA), na Educação a Distância (EAD) ou nos cursos presenciais, têm se mostrado vantajosos como modelo de interação, respeito ao ritmo individual, disponibilidade de recursos educacionais e atendimento a um grande número de alunos. Contudo, a evasão nessa modalidade de ensino [Queiroga et al. 2017] é significativa e entre os motivos destacam-se: econômico, vocacional e institucional, que se refere à adoção de modelos de aprendizagem inadequados e desmotivação dos alunos. Os estilos de aprendizagem (EA) se referem às predisposições das pessoas no processo de ensino e aprendizagem, ou seja, às preferências dos alunos em relação à recepção e processamento de informação [Felder 
VIII Congresso Brasileiro de Informática na Educação (CBIE 2019)

Anais do XXX Simpósio Brasileiro de Informática na Educação (SBIE 2019)

and Silverman 1988]. No contexto da evasão escolar, EA são usados como forma de reconhecer, precocemente, alunos com risco de desistência, visto que a evasão pode ser associada a uma sequência de comportamentos distintos às preferências dos alunos [Gohil et al. 2018]. Os EA e a AS são temas de interesse de pesquisadores por favorecerem a personalização de sistemas e-learning [Faria et al. 2017; Frozza et al. 2011]. Esses sistemas procuram atender as necessidades dos alunos, recomendando material motivacional ou didático que melhor se adequa às suas preferências.

Este artigo é parte integrante do projeto BROAD que inclui a aplicação de Sistemas de Recomendação e Ecossistemas de Software na Educação [Pereira et al. 2018]. Este trabalho descreve a arquitetura SASys, baseada em uma rede de frames, que tem como objetivo principal determinar o sentimento do autor em textos e ampliar a assertividade da detecção do estado emocional do mesmo. Ao final, um sistema de recomendação baseado no estado emocional do aluno e do seu estilo de aprendizagem envia mensagens motivacionais para mitigar a evasão. A condução dessa pesquisa incluiu uma revisão sistemática da literatura sobre estilos de aprendizagem, e outra sobre análise de sentimento, a definição e implementação de uma arquitetura e um estudo de caso no âmbito de uma disciplina no ambiente virtual Moodle.

O artigo está organizado da seguinte forma: na seção 2 são apresentados os principais conceitos e na seção 3 os trabalhos relacionados. A arquitetura é apresentada na seção 4, na seção 5 é descrito o estudo de caso e na seção 6 as considerações finais.

\section{Pressupostos Teóricos}

Estilos de Aprendizagem são mecanismos utilizados para se obter as preferências do aluno. Eles se referem aos gostos ou escolhas em relação à recepção e processamento de informação [Felder and Silverman 1988]. São, normalmente, agrupados em Modelos de Estilos de Aprendizagem, que classificam os alunos de acordo com escalas predeterminadas. Os modelos mais citados na literatura são os de Kolb, Felder e Silverman e VARK [Nascimento et al. 2017; Valaski et al. 2011], identificados na revisão da literatura realizada ${ }^{1}$. Eles são comumente utilizados no contexto de e-learning para melhorar o desempenho de sistemas adaptativos, pois é possível traçar, através deles, os perfis dos alunos [Valaski et al. 2011].

Informações sobre o estado emocional do aluno agregam valor ao seu perfil, visto que o mesmo pode afetar o desempenho acadêmico [Liting Xu et al. 2008] [Vivian et al. 2016]. Para [Silva et al. 2016], cada aluno tem sua maneira própria de receber e analisar as informações e é importante conhecer os EA para entender métodos e técnicas de ensino que funcionam melhor em cada contexto. Combinar o estado emocional e o estilo de aprendizagem do aluno pode potencializar o aproveitamento acadêmico.

Análise de Sentimento (AS) pode ser definida como um estudo computacional de opiniões, sentimentos e emoções expressas em um texto [Altrabsheh et al. 2013] com relação a produtos, serviços, organizações, indivíduos, etc. [İskender and Bat1 2015]. $\mathrm{Na}$ educação é usada na compreensão do processo de ensino e aprendizagem e identificação de sentimentos. Os negativos podem levar ao abandono das disciplinas e cursos. A AS abrange soluções que vão de técnicas Lexicais a algoritmos de Aprendizado de Máquina. As técnicas lexicais buscam por palavras carregadas de

1https://drive.google.com/file/d/1kLM8saQPq2zS1KhGW0BYP8FyGVG7mbp3/view?usp=sharing 
VIII Congresso Brasileiro de Informática na Educação (CBIE 2019)

Anais do XXX Simpósio Brasileiro de Informática na Educação (SBIE 2019)

sentimento através de técnicas de Processamento de Linguagem Natural (PLN) e Análise de Texto, e, normalmente, são baseadas em dicionários ou corpus de palavras [Altrabsheh et al. 2014] [Hemmatian and Sohrabi 2017].

A FrameNet Brasil é um laboratório de Linguística Computacional que tem como objetivo desenvolver soluções computacionais para problemas de Processamento de Linguagem Natural, usando Semântica de Frames [Salomão 2009]. Também chamada de semântica da compreensão, consiste em dar significado às palavras através de frames semânticos [Fillmore et al. 2003], que são estruturas conceituais que descrevem situações, objetos, ou eventos, juntamente com seus participantes e adereços. Atualmente a FrameNet Brasil possui mais de 13.000 unidades lexicais distribuídas pelos 1.200 frames e, neste trabalho, ela é usada como uma solução lexical para a Análise de Sentimentos, pois, além de possuir um corpus considerável, contém semântica associada às palavras.

\section{Trabalhos Relacionados}

Os autores de [Liu et al. 2013] apresentam um sistema de recomendação baseado em análise de sentimento de comentários on-line. Este sistema analisa a diferença entre a classificação e a opinião do usuário para identificar as suas preferências. A AS é abordada como um problema de extração de características, que ajuda a inferir os itens a serem recomendados. A nossa proposta se assemelha no uso da AS para melhorar a recomendação, porém, ela é adotada para identificar o momento da recomendação que possa retardar a evasão. Além disso, a preferência do estudante é determinada pelo EA.

Os autores [López et al. 2016] apresentam a arquitetura EmoRemSys, que é um sistema de recomendação educacional baseado em computação afetiva. A arquitetura usa a API SkyBiometry para identificação de emoções através de expressões faciais e o filtro colaborativo para a recomendação de conteúdo. O nosso trabalho é semelhante no que tange ao domínio educacional e ao uso da AS para determinar o instante da recomendação, porém, a AS é feita em textos extraídos do AVA e da rede social, por serem fontes menos invasivas e com maior riqueza emocional, além de não ser dependente de outro recurso computacional, como uma webcam por exemplo.

\section{Arquitetura SASYS}

SASys (Sentiment Analisys System) é uma arquitetura que detecta o estado emocional do autor de um texto, num determinado momento, através da orientação semântica das palavras. Ela usa abordagens de AS e de uma rede de frames polarizada, derivada da FrameNet Brasil, para a caracterização do sentimento. A SASys tem como objetivo principal fazer o uso da rede de frames para determinar o sentimento do autor em textos e ampliar a assertividade da detecção do seu estado emocional.

O fluxo de detecção do estado emocional se inicia com a extração de dados do autor de alguma fonte. Os dados são usados tanto para AS como para a definição do perfil. Depois de coletados, são aplicadas técnicas de pré-processamento aos textos, como remoção de sinal de pontuação ou quebra de linha. Em seguida os textos são submetidos à rede complexa formada pelos frames da FrameNet Brasil, para a identificação das palavras com polaridade. Após essa etapa, a orientação semântica do texto é dada a partir do resultado de alguma das abordagens de AS (Lexical, 
VIII Congresso Brasileiro de Informática na Educação (CBIE 2019)

Anais do XXX Simpósio Brasileiro de Informática na Educação (SBIE 2019)

Aprendizado de Máquina ou Híbrida). O fluxo termina com a identificação do estado emocional do autor do texto.

A SASys é uma arquitetura composta por 3 camadas, a saber:

Extração de dados: responsável por identificar os dados do autor do texto e coletar os mais relevantes para a AS e composição do perfil emocional. Os dados explícitos englobam dados de perfil, como nome, idade e idioma, e os de contexto que se referem à localização, dispositivo usado, entre outros. Os dados implícitos carregam maior valor semântico, e podem ser dados de interação, acesso, feedback e textos.

Análise de sentimentos: depois de coletar os dados, é necessário o uso de alguma técnica de pré-processamento, antes de submetê-los a qualquer tipo de solução de AS. O pré-processamento é a primeira etapa da AS e é empregado para limpar o texto inicial de dados não relevantes. Ele tem o propósito de modelar os dados de acordo com a entrada do algoritmo usado, além de reduzir a inconsistência, viabilizando o aumento da acurácia do método [Altrabsheh et al. 2014]. As frases são então passadas à rede de frames polarizada, que identifica os frames evocados pelas unidades lexicais e retorna suas polaridades: positiva, negativa ou neutra. A saída da rede de frames pode ser usada como parâmetro de entrada do método de AS escolhido. O sentimento geral pode ser calculado tendo em conta os sentimentos individuais das unidades lexicais e os transformadores de valência presentes nas frases. Os transformadores de valência são termos que alteram a polaridade da palavra mais próxima que possua sentimento, como intensificadores (muito, absolutamente, demasiado, sinais de exclamação e interrogação repetidos), redutores (pouco, menos, quase, apenas) e negações (não, jamais, nunca).

Identificação do estado emocional: a identificação da polaridade do texto é uma informação relevante que, combinada com outros dados, pode revelar o estado emocional do autor e até traçar um possível perfil emocional do mesmo. Além de saber como o autor se sentia enquanto escrevia o texto, distinguir a sua idade, gênero, localização, condição social, horário que escreveu, com quem interagiu, dispositivo que usou, entre outros, pode agregar valor semântico à análise [Altrabsheh et al. 2013] [Liting Xu et al. 2008].

$\mathrm{Na}$ arquitetura proposta, a detecção do estado emocional é feita depois de gerado o sentimento geral do texto, usando também os dados de perfil e de contexto. Esses dados podem ser submetidos a métodos estatísticos e o resultado obtido, combinado com a AS, fornecem o status do autor, no momento da escrita, que pode ser utilizado para compor o perfil do usuário em uma aplicação ou até para recomendação de material personalizado. Neste trabalho foram definidas três polaridades: positiva palavras que evocam um sentimento positivo, negativa - palavras que evocam um sentimento negativo, e neutra - palavras que não fazem um julgamento prévio. A polaridade neutra foi usada porque a FrameNet Brasil possui uma quantidade considerável de unidades lexicais que evocam cenários sem sentimentos. A solução proposta foi implementada utilizando a linguagem de programação Python e a biblioteca Network $X^{2}$ que permite criar, manipular e usar funções de redes complexas.

\footnotetext{
${ }^{2}$ https://networkx.github.io/
} 
VIII Congresso Brasileiro de Informática na Educação (CBIE 2019)

Anais do XXX Simpósio Brasileiro de Informática na Educação (SBIE 2019)

Depois de obtida a rede polarizada, a mesma foi armazenada no banco de dados $\mathrm{Neo}^{3} j^{3}$, que permite consultas em grafos.

\section{Estudo de Caso}

O estudo de caso [Dresch et al. 2015] é adequado para a avaliação da SASys por se tratar de uma pesquisa empírica, realizada em um contexto real, que deseja investigar a adequação da arquitetura ao modelo de detecção de estado emocional. O estudo de caso foi conduzido no contexto de $\mathrm{EaD}$ com o propósito de avaliar a arquitetura SASys no reconhecimento do estado emocional do aluno, através do uso da rede de frames.

Ele foi realizado em uma turma da disciplina de Metodologia de Pesquisa Científica e Educacional, ofertada na modalidade a distância na Universidade Federal de Juiz de Fora, no primeiro semestre de 2018. A disciplina foi ministrada a 58 alunos com apoio do Moodle. As atividades foram disponibilizadas semanalmente e executadas em grupo, com um número máximo de 3 participantes. Esta característica foi determinante na análise dos dados, pois os grupos eram, majoritariamente, liderados por um único aluno, que se encarregava de enviar as atividades. Cada atividade possuía um fórum de dúvidas específico, usado para a análise de sentimento. As mensagens dos alunos eram agrupadas pelos fóruns e, conseqüentemente, em semanas, por esse motivo definiu-se janelas de tempo de 7 dias nas análises de interação com o ambiente de aprendizagem.

A base de dados para a AS consistiu dos comentários coletados dos fóruns, visto que os alunos não possuíam uma rede social específica. Essa base de dados foi inicialmente rotulada por dois professores, um com mais de 15 anos e outro com 6 anos de experiência em EaD, com o objetivo de comparar a expertise humana em identificar alunos com sentimentos negativos, no caso com possibilidade de evasão da disciplina, com a abordagem proposta neste trabalho. Neste estudo de caso, a atenção foi canalizada no sentimento negativo, pois se pressupõe que ele seja um dos elementos que contribui no aumento da propensão à evasão escolar. Num segundo momento, a base de dados passou pela avaliação de um lingüista da FrameNet Brasil.

\subsection{Etapas do estudo de caso}

Coleta de dados: A arquitetura foi instanciada para o estudo de caso no Moodle (Figura 1). Os alunos responderam a um questionário on-line para identificar o dispositivo mais usado e preferências da forma de apresentação do conteúdo (texto, áudio ou vídeo), visando a recomendação de mensagens a partir da identificação do perfil motivacional. Os dados implícitos foram coletados sobre os acessos dos alunos e extraídos dos textos dos fóruns. Os fóruns foram escolhidos por conterem mensagens com teor emocional e por serem o veículo de comunicação entre os estudantes, o professor e o tutor. Foram consideradas apenas as frases escritas pelos alunos e ignoradas todas as respostas do professor e tutor. Após o pré-processamento foram selecionadas 27 mensagens de 10 fóruns.

Análise de Sentimento: A rotulação das mensagens ocorreu em duas etapas. Na primeira predominou a expertise de dois professores e na segunda um linguista especialista da FrameNet Brasil, utilizou de técnicas para detecção de sentimento. Os docentes qualificaram as frases em uma das três categorias: Positivas (elogios, detecção de inconsistência, colaboração com os outros colegas, mensagem motivacional,

\footnotetext{
${ }^{3}$ https://neo4j.com/
} 
VIII Congresso Brasileiro de Informática na Educação (CBIE 2019)

Anais do XXX Simpósio Brasileiro de Informática na Educação (SBIE 2019)

contribuição no conteúdo), Neutras (dúvidas de execução, mensagem social ou de interação) e Negativas (dúvidas de conteúdo, dificuldades de entendimento, relatos de problemas pessoais e sentimento de frustração ou de não entendimento).

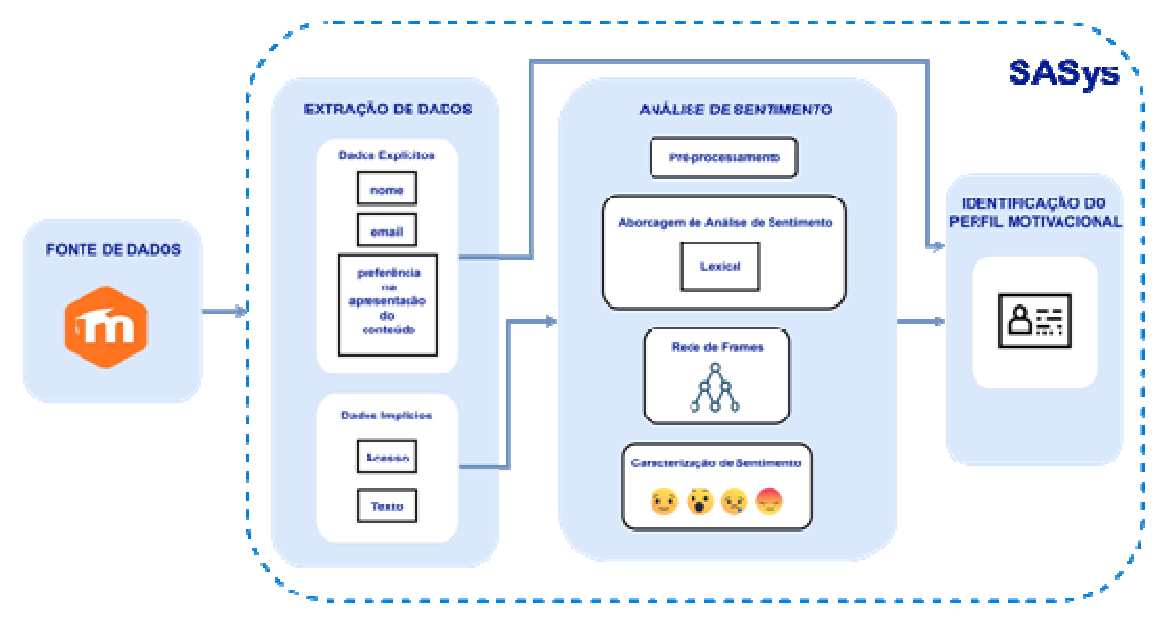

Figura 1 - Arquitetura SASys para ambientes virtuais de aprendizagem

Das 27 mensagens, 2 foram classificadas como positivas, 13 como negativas e 12 neutras e a seguir tem um exemplo de cada categoria. Somente os alunos que publicaram nos fóruns tiveram seus perfis motivacionais detectados. Dentre todos os alunos, sete foram responsáveis pela geração de todo conteúdo negativo, sendo que três deles tiveram o sentimento recorrente, ou seja, publicaram mais de uma vez mensagens com essa polaridade.

\begin{tabular}{l|l}
\hline Frase & Rótulo \\
\hline $\begin{array}{l}\text { A etapa vence no dia 29, correto? Pergunto pois dentro do link da tarefa fala que vence no dia } \\
22, \text { e na parte da etapa fala que vence no dia } 29\end{array}$ & Positiva \\
\hline $\begin{array}{l}\text { Estou fazendo as tarefas, mas estou muito em dúvida se estou indo pelo caminho certo, tenho } \\
\text { muitas dificuldades neste trabalho }\end{array}$ & Negativa \\
\hline Boa tarde Professora, a tarefa sobre o objetivo dessa semana pode ser feita em meia lauda? & Neutra \\
\hline
\end{tabular}

A AS foi feita considerando as polaridades retornadas pela rede de frames com os transformadores de valência. Os comentários foram divididos em sentenças, depois geradas e somadas as suas polaridades, para a obtenção do sentimento geral do texto. A operação de soma foi uma operação binária onde o sentimento geral do comentário completo era determinado pelas seguintes condições:

- $\quad S e$ as duas frases têm sentimentos iguais, então sentimento recorrente;

- Se uma das frases tem sentimento negativo, então sentimento negativo;

- Se uma das frases tem sentimento neutro, então o outro sentimento.

O exemplo a seguir ilustra o processo de detecção da unidade lexical com polaridade encontrada na frase e a geração do sentimento geral do comentário. A rede de frames conseguiu uma acurácia de $62 \%$, com precisão de $46 \%$ e recall de $42 \%$, valores razoáveis para uma abordagem lexical [Hemmatian and Sohrabi 2017].

\begin{tabular}{l|l|l|l}
\hline \multicolumn{1}{c|}{ Comentário } & \multicolumn{1}{c|}{$\begin{array}{c}\text { Unidade Lexical } \\
\text { evocada (polaridade) }\end{array}$} & $\begin{array}{c}\text { Polaridade } \\
\text { parcial }\end{array}$ & $\begin{array}{c}\text { Sentimento } \\
\text { (rede de frames) }\end{array}$ \\
\hline $\begin{array}{l}\text { Tive um infortúnio não consegui enviar a tarefa dentro } \\
\text { da hora solicitada (23:55)... Agora são 23:58h... }\end{array}$ & $\begin{array}{l}\text { tive(neutra), não(negação } \\
\text { alcançada) consegui(positiva } \\
\text { Conforme a orientação no guia do aluno as atividades } \\
\text { com entrega no atraso são avaliadas com desconto de } \\
\text { 50\% no valor da nota, porém o link para enviar a } \\
\text { atividade não se encontra disponível. Como faço pra } \\
\text { poder enviar a tarefa solicitada depois do prazo? }\end{array}$ & $\begin{array}{l}\text { alcançada) se(redutor } \\
\text { alcançado) disponível } \\
\text { positiva }->\text { negativa), } \\
\text { poder(positiva) }\end{array}$ & Negativa \\
\hline
\end{tabular}


VIII Congresso Brasileiro de Informática na Educação (CBIE 2019)

Anais do XXX Simpósio Brasileiro de Informática na Educação (SBIE 2019)

Com o objetivo de aprimorar a identificação de sentimento a rede de frames polarizada foi submetida à avaliação de um lingüista especialista da FrameNet Brasil. Algumas melhorias foram sugeridas:

- Aumento do alcance dos transformadores de valência: a estratégia usada era baseada no 3-gram [Dey et al. 2017], onde 3 era o número de palavras alcançadas por negações, intensificadores ou redutores de valência. O seu alcance foi estendido até o sinal de pontuação mais próximo, abrangendo e influenciando todas as outras palavras no caminho.

- Verbos que expressam "dever" têm sentimento negativo e os que expressam "possibilidade" têm sentimento positivo: a distinção foi feita devido aos verbos dever, precisar, ter, poder, escolher, entre outros, que estavam sendo tratados como palavras neutras.

- As aspas representam citação e não devem ser usadas na análise de sentimento: na nova estratégia, foi definido o número 4 como a quantidade mínima de palavras para que a sentença fosse considerada uma citação.

A AS foi refeita nos textos dos alunos, e houve alteração positiva nos valores das métricas acurácia e precisão, evidenciando que a expansão do alcance dos redutores melhorou o desempenho da abordagem.

Identificação do perfil motivacional: $\mathrm{O}$ resultado da AS foi usado junto com as interações dos alunos no AVA para gerar os perfis motivacionais, baseado no estado emocional dos mesmos. Os logs dos alunos foram coletados o que permitiu uma análise estatística mais refinada buscando compreender melhor os seus comportamentos. A Figura 2 mostra a distribuição das interações de todos os alunos por semana utilizando Boxplot. Todos os gráficos têm o mínimo em zero por existir, pelo menos, um aluno que não interagiu com o ambiente na respectiva semana. A grande maioria dos outliers representa os alunos líderes dos grupos. A partir da Semana 7, o valor da mediana decaiu, dando indícios do aumento de alunos com pouca interação e, consequentemente, da existência de alunos com risco de evasão. O resultado da AS e das análises estatísticas dos dados coletados gerou 4 estados emocionais e, consequentemente, identificou 4 categorias nos Perfis Motivacionais:

- PM1 - Entusiasmo - Alunos com um número grande de ações no AVA. Estes alunos tendem a ser mais participativos, a realizar todas as atividades propostas no curso e possuírem sentimentos positivos ou neutros. A média de interação dos alunos deste grupo foi de 20 ações por semana;

- PM2 - Moderação e Dúvida - Alunos com um número razoável de ações no AVA. Este grupo abrange dois estados emocionais: Moderação, estudantes que frequentam a disciplina com regularidade, tendem a realizar boa parte das atividades propostas e raramente comentam em fóruns; e Dúvida, estudantes que interagem com o fórum reportando dúvidas de execução de tarefa ou de conteúdo. De forma generalizada, alunos deste grupo apresentam sentimentos neutros e negativos. A média de interação dos alunos deste grupo foi entre 10 e 19 ações por semana;

- PM3 - Desânimo - Alunos com pouca interação (alerta de evasão). Estes alunos tendem a ficar vários dias sem acessar o ambiente educacional e a realizar um número reduzido de atividades da disciplina. Seus comentários são de desorientação e frustração, indicando o sentimento negativo. A média de interação deste grupo foi menos de 10 ações por semana;

- PM4 - Alunos com interação quase nula (iminência de evasão) - Alunos que interagiram com o AVA e que, depois de um período de tempo, o seu número de ações ficou nulo e, por este motivo, não possuem comentários para a AS. A média de interação dos alunos deste grupo foi menos de 5 ações por semana.

Os PM1 e PM2 com sentimentos negativos foram considerados alunos em estado de alerta porque, apesar de se mostrarem interessados com a disciplina, possuem inquietações que podem levá-los a desmotivação. Os PM3 e PM4 contêm os alunos que necessitam de uma atenção especial por parte do professor, por serem alunos que têm maior probabilidade de desistir do curso. A Figura 3 mostra a distribuição dos alunos nos respectivos perfis motivacionais. Cada boxplot foi gerado com as médias de 
VIII Congresso Brasileiro de Informática na Educação (CBIE 2019)

Anais do XXX Simpósio Brasileiro de Informática na Educação (SBIE 2019)

interação dos alunos. As medianas dos boxplots foram usadas como o limite motivacional de cada perfil, sendo PM1 $=22, \mathrm{PM} 2=14, \mathrm{PM} 3=6$ e PM4 $=3$. Os alunos com média de ação abaixo da mediana, com sentimento negativo recorrente bem como os pertencentes aos PM3 e PM4, foram considerados desmotivados e com risco de evasão.
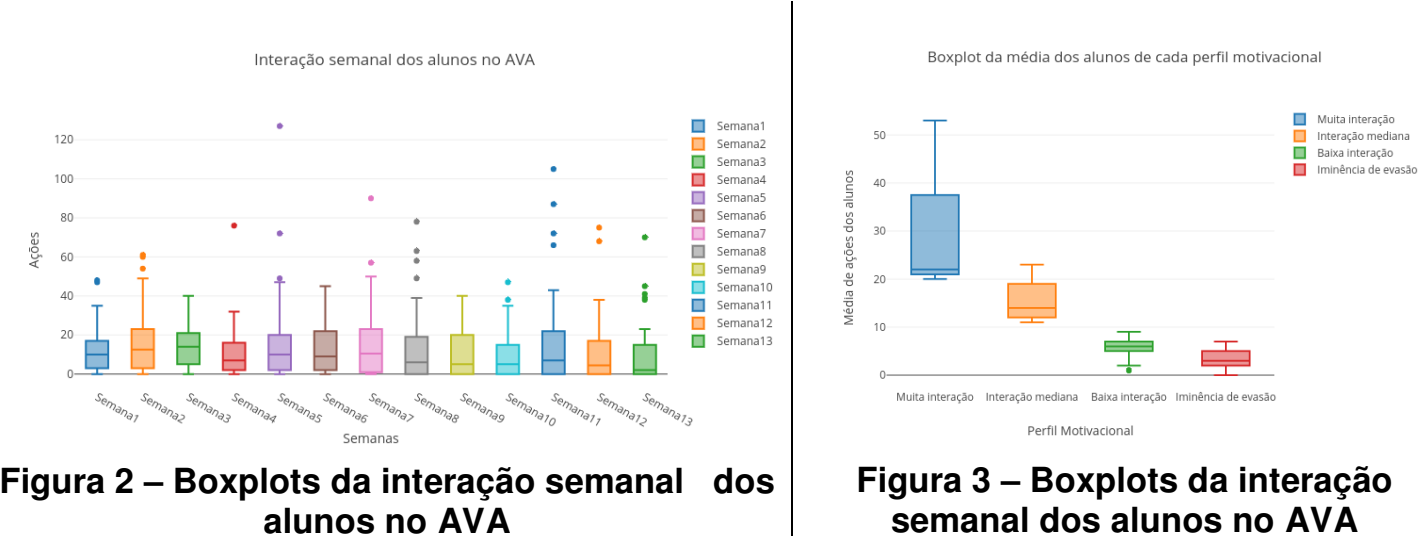

Figura 3 - Boxplots da interação semanal dos alunos no AVA

\subsection{Recomendação de mensagens a partir do Perfil Motivacional}

A partir do perfil motivacional dos alunos identificados como desmotivados, foi desenvolvido um sistema de recomendação para envio de mensagens aderentes às suas preferências buscando reverter a tendência à evasão. As mensagens foram geradas em três tipos de mídias: vídeo, texto e áudio (Figura 4).
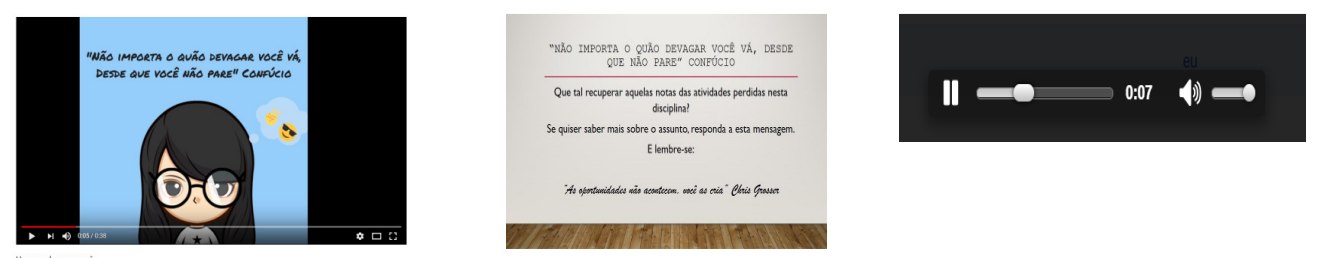

Figura 4 - Mídias das mensagens motivacionais

Neste estudo de caso foram identificados 5 alunos com indícios de risco de evasão, com rendimento acadêmico baixo na disciplina, e que responderam ao questionário sobre perfil. Eles receberam as mensagens motivacionais e as responderam em seu ambiente Moodle. Foi possível coletar feedback positivo, exceto de um do PM3, que não respondeu à mensagem. Os alunos se mostraram interessados em recuperar as atividades não realizadas, voltaram a interagir no Moodle e enviaram as atividades para correção. O feedback dos alunos e o envio das atividades dão indícios de que a recomendação de conteúdo motivacional é uma forma de redução do risco de evasão.

\subsection{Análise dos resultados}

Neste estudo de caso foi possível fazer a análise de sentimento em textos de alunos de uma disciplina on-line e coletar suas interações para identificar seus perfis motivacionais, identificando aqueles com risco de evasão. Os textos dos alunos foram retirados de fóruns de dúvidas o que implicou em um número considerável de frases negativas e neutras. Os textos dos alunos foram rotulados por professores com experiência em $\mathrm{EaD}$ o que proporcionou um desempenho razoável para a abordagem lexical proposta neste trabalho. A avaliação por um especialista da FrameNet Brasil, que sugeriu melhorias, resultou num desempenho ainda melhor da abordagem. Os resultados iniciais motivaram o uso da FrameNet como um dicionário de sentimentos 
VIII Congresso Brasileiro de Informática na Educação (CBIE 2019)

Anais do XXX Simpósio Brasileiro de Informática na Educação (SBIE 2019)

que pode ser usado em mais de uma língua. Entretanto, outras avaliações devem ser conduzidas para a consolidação da proposta da arquitetura.

\section{Considerações Finais}

Este artigo apresentou a arquitetura SASys, instanciada no contexto da educação, capaz de identificar o perfil motivacional do aluno através de AS. A proposta foi avaliada através de um estudo de caso em um ambiente real de utilização onde os estilos de aprendizagem e os níveis motivacionais foram identificados, a análise de sentimentos foi feita nos comentários dos fóruns de dúvidas e conteúdos motivacionais foram recomendados. Os feedbacks dos alunos e a interação no AVA dão indícios da viabilidade da proposta na busca pela redução da evasão em cursos EAD. A análise de sentimento usando a rede de frames se mostrou viável obtendo uma precisão de $65 \%$ e recall de $68 \%$, que são valores que estão dentro do esperado, para uma abordagem lexical. Como trabalho em andamento destacamos a ampliação do material motivacional e como trabalhos futuros cabe avaliar a arquitetura sob outras perspectivas, buscando, principalmente, identificar quais emoções (alegria, sarcasmo, entusiasmo, etc.) estão associadas a determinados Estilos de Aprendizagem, e como as mudanças emocionais podem sugerir alterações de preferências dos alunos.

Agradecimentos: Agradecemos à FAPEMIG, CNPq, Capes e UFJF pelo financiamento e suporte ao Projeto BROAD e à Framenet Brasil pela colaboração nesta pesquisa.

\section{Referências}

Altrabsheh, N., Cocea, M. and Fallahkhair, S. (2014). Sentiment Analysis: Towards a Tool for Analysing Real-Time Students Feedback. In 2014 IEEE 26th International Conference on Tools with Artificial Intelligence. IEEE. http://ieeexplore.ieee.org/lpdocs/epic03/wrapper.htm?arnumber=6984506, November.

Altrabsheh, N., Gaber, M. M. and Cocea, M. (2013). SA-E: Sentiment analysis for education. Frontiers in Artificial Intelligence and Applications. v. 255p. 353-362.

Bamakan, S. M. H., Nurgaliev, I. and Qu, Q. (2019). Opinion leader detection: A methodological review. Expert Systems with Applications, v. 115, p. 200-222.

Dey, A., Jenamani, M. and Thakkar, J. J. (2017). Lexical TF-IDF: An n-gram Feature Space for Cross-Domain Classification of Sentiment Reviews. Springer, Cham. p. $380-386$.

Dresch, A., Lacerda, D. P. and Antunes Jr, J. A. V. (2015). Design Science Research. Design science research: A method for science and technology advancement., Porto Alegre, Bookman, p 204.

Faria, A. R., Almeida, A., Martins, C., et al. (2017). A global perspective on an emotional learning model proposal. Telematics and Informatics, v. 34, n. 6, p. 824-837.

Felder Richard M., N. C. S. U. and Silverman Linda K., I. for the S. of A. D. (1988). Learning and Teaching Styles in Engineering Education. Engr. Education. http://www.ncsu.edu/felder-public/ILSpage.html, accessed on Jul 42019.

Fillmore, C. J., Johnson, C. R. and Petruck, M. R. L. ( 2003). Background to Framenet. International Journal of Lexicography, v. 16, n. 3, p. 235-250.

Frozza, R., Da Silva, A. A. K., Schreiber, J. N. C., et al. (2011). Agentes Pedagógicos Emocionais atuando em um Ambiente Virtual de Aprendizagem. 
VIII Congresso Brasileiro de Informática na Educação (CBIE 2019)

Anais do XXX Simpósio Brasileiro de Informática na Educação (SBIE 2019)

RENOTE, v. 9, n. 1.

Gohil, S., Vuik, S. and Darzi, A. (2018). Sentiment Analysis of Health Care Tweets: Review of the Methods Used. JMIR Public Health and Surveillance, v. 4, n. 2, p. e43.

Hemmatian, F. and Sohrabi, M. K. (2017). A survey on classification techniques for opinion mining and sentiment analysis. Artificial Intelligence Review, H, [s. 1.].

İskender, E. and Bat1, G. B. (2015). Comparing Turkish Universities Entrepreneurship and Innovativeness Index's Rankings with Sentiment Analysis Results on Social Media. Procedia - Social and Behavioral Sciences, v. 195, p. 1543-1552.

Liting $\mathrm{Xu}$, Yongzhao Zhan and Miao Zhu (2008). A guide based on situation and emotion analysis in ontology-based E-Learning system. In 2008 3rd IEEE Conference on Industrial Electronics and Applications. . IEEE. http://ieeexplore.ieee.org/document/4582950/, June.

Liu, H., He, J., Wang, T., Song, W. and Du, X. (2013). Combining user preferences and user opinions for accurate recommendation. Electronic Commerce Research and Applications, v. 12, n. 1, p. 14-23.

López, M. B., Montes, A. J. H., Ramírez, R. V., et al. (2016). EmoRemSys: Sistema de recomendación de recursos educativos basado en detección de emociones. RISTI Revista Ibérica de Sistemas e Tecnologias de Informação, n. 17, p. 80-95.

Lu, J., Wu, D., Mao, M., Wang, W. and Zhang, G. (2015). Recommender system application developments: A survey. Decision Support Systems, v. 74, p. 12-32.

Nascimento, P. Do, Barreto, R., Primo, T., Gusmão, T. and Oliveira, E.. Recomendação de Objetos de Aprendizagem baseada em Modelos de Estilos de Aprendizagem: Uma Revisão Sistemática da Literatura. . http://www.brie.org/pub/index.php/sbie/article/view/7550, acessado 27/10/2017.

Pereira, C. K., Campos, F., Ströele, V., David, J. M. N. and Braga, R. (2018). BROAD-RSI - educational recommender system using social networks interactions and linked data. Journal of Internet Services and Applications, v. 9, n. 1, p. 7.

Queiroga, E., Cechinel, C. and Araújo, R. (2017). Predição de estudantes com risco de evasão em cursos técnicos a distância. http://www.brie.org/pub/index.php/sbie/article/view/7686, acessado 27/10/2019.

Salomão, M. M. M. (2009). FrameNet Brasil: um trabalho em progresso. Calidoscópio, v. 7, n. 3, p. 171-182.

Silva, Z., Ferreira, L. and Pimentel, A. (2016). Modelo de Apresentação Adaptativa de Objeto de Aprendizagem baseada em Estilos de Aprendizagem. . http://www.brie.org/pub/index.php/sbie/article/view/6757, acessado 7/11/2016.

Valaski, J., Malucelli, A. and Reinehr, S. (2011). Revisão dos Modelos de Estilos de Aprendizagem Aplicados à Adaptação e Personalização dos Materiais de Aprendizagem. In Anais do Simpósio Brasileiro de Informática na Educação. http://www.br-ie.org/pub/index.php/sbie/article/view/1843.

Vivian, R., Falkner, K., Falkner, N. and Tarmazdi, H. (17 feb 2016). A Method to Analyze Computer Science Students' Teamwork in Online Collaborative Learning Environments. ACM Transactions on Computing Education, v. 16, n. 2, p. 1-28. 